PROCEEDINGS OF THE

AMERICAN MATHEMATICAL SOCIETY

Volume 93, Number 2, February 1985

\title{
A THEOREM ON THE TENSION FIELD
}

\author{
TH. KOUFOGIORGOS AND CH. BAIKOUSSIS
}

\begin{abstract}
Suppose $M$ and $N$ are complete Riemannian manifolds; $M$ with Ricci curvature bounded from below and $N$ with sectional curvature bounded from above by a constant $K_{0}$. Let $f: M \rightarrow \cdot N$ be a smooth map such that $f(M) \subset B_{R}$, where $B_{R}$ is a normal ball in $N$ and furthermore $R<\pi / 2 \sqrt{K_{0}}$ if $K_{0}>0$. If the energy density $e(f)$ is bounded below by a positive constant, then there is a point $P \in M$ such that the tension field $\tau(f)$ at $P$ is different from zero.
\end{abstract}

1. Introduction. Let $M$ be a complete Riemannian manifold with Ricci curvature bounded below by a constant and $N$ be a complete Riemannian manifold with sectional curvature $K$ bounded above by a constant $K_{0}$. Suppose $f: M \rightarrow N$ is a smooth map such that $f(M)$ lies in a normal ball $B_{R}$ of radius $R$. From now on, we will moreover suppose that $R<\pi / 2 \sqrt{K_{0}}$ if $K_{0}>0$. It is well known that there can be harmonic maps $f: M \rightarrow N$ with $f(M) \subset B_{R}$. For example if $M$ is the hyperbolic half plane and $N$ is the euclidean space $E^{2}$ then the map $f(z)=(z-i) /(z+i)$, $z \in C$, is harmonic and $f(M)$ is contained in the unit disk $D^{2}$ of $E^{2}$. We remark that there are points $(z \rightarrow \infty)$ on $M$ where the energy density $e(f)$ of $f$ tends to zero. This and all the examples known to us suggested considering whether for any harmonic map $f: M \rightarrow N$ there must be sequences of points on $M$ where $e(f)$ tends to zero. The answer is given in the following theorem.

THEOREM. Let $f: M \rightarrow N$ be a smooth map such that $f(M) \subset B_{R}(R<$ $\pi / 2 \sqrt{K_{0}}$, if $\left.K_{0}>0\right)$. If $e(f)$ is bounded below away from zero then there exists a point $P \in M$ such that the tension field $\tau(f)$ at $P$ is different from zero.

2. Preliminaries. We denote $\langle,\rangle_{M}$ and $\langle,\rangle_{N}$ the metrics of $M$ and $N$ respectively. Given a smooth map $f: M \rightarrow N$ the inverse image $f^{*}\left(\langle,\rangle_{N}\right)$ considered as a 2 -form on $M$ is positive semidefinite and is called the first fundamental form of $f$. The induced vector bundle $E=f^{-1} T N \rightarrow M$ has a Riemannian structure $\langle,\rangle_{E}$ induced from that of $N$. Thus if $\nabla$ and $\bar{\nabla}$ are the Riemannian connections in $M$ and $N$ respectively, then for any $Y \in T M$ and for any section $w \in \beta(E)$, where $\beta(E)$ is the set of sections of the bundle $E$, the induced Riemannian connection $\overline{\bar{\nabla}}$ in $E$ is defined as follows. We consider a local frame field $p_{a}$ in $T N$ so that for $w \in \beta(E)$ there are real functions $h^{a}$ in a chart of $M$ with $w=\sum_{a} h^{a} p_{a}$. Then we set

$$
\overline{\bar{\nabla}}_{Y} w=\sum_{a}\left\{\left(Y h^{a}\right) p_{a}+h^{a} \bar{\nabla}_{f_{*} Y} p_{a}\right\}
$$

We note that the differential $d f$ of $f$, for which we write $f_{*}$, can be interpreted as a $E$-valued 1-form of $M$ i.e., $f_{*} \in \beta\left(T^{*} M \otimes E\right)$, where $T^{*} M$ is the dual bundle

Received by the editors August 10, 1983 and, in revised form, March 26, 1984.

1980 Mathematics Subject Classification. Primary 53C99; Secondary 58E20, 35J60.

(c) 1985 American Mathematical Society $0002-9939 / 85 \$ 1.00+\$ .25$ per page 
of the tangent bundle $T M$. The bundle $T^{*} M \times E \rightarrow M$ inherits a Riemannian structure from those of $M$ and $E$. Thus the norm $\left\|f_{*}\right\|$ of $f_{*}$ is defined by $\left\|f_{*}\right\|^{2}=$ $\sum_{i}\left\langle f_{*} Y_{i}, f_{*} Y_{i}\right\rangle_{N}$, where $Y_{i}$ is an orthonormal basis in $M$. $\left\|f_{*}\right\|^{2}$ is known as the energy density of $f$ and it is denoted by $e(f)$. The covariant differential $\tilde{\nabla} f_{*} \in$ $\beta\left(T^{*} M \otimes T^{*} M \otimes E\right)$ is called the second fundamental form of $f$ and it is denoted by $L$. We can see that for vector fields $X, Y$ on $M$ it is valid: $L(X, Y)=\overline{\bar{\nabla}}_{X}\left(f_{*} Y\right)-$ $f_{*}\left(\nabla_{X} Y\right)$. The tension field $\tau(f)$ of $f$ is a section of the vector bundle $E$ and it is defined as the trace of the second fundamental form of $f$. If $\tau(f) \equiv 0$, then $f$ is called harmonic. For the above definitions see [2].

We are going to use the following three lemmas.

LEMMA 1. Let $\Phi: M \rightarrow R$ be a smooth function having an upper bound. Then for any point $P \in M$ and for any $\varepsilon>0$, there exists a point $q \in M$ depending on $P$, such that $\Phi(q) \geq \Phi(P),\|\operatorname{grad} \Phi\|(q)<\varepsilon$ and the Laplacian $(\Delta \Phi)(q)<\varepsilon$.

LEMMA 2. Suppose $x_{0}$ and $x$ are points of $N$ so that $x$ does not lay in the cut locus of $x_{0}$. Let $\gamma:[0,1] \rightarrow N$ be the minimizing geodesic segment connecting $x_{0}$ with $x$ parametrized proportionally to the arc length. Take a positive number a. For any Jacobi field $X$ along $\gamma$ which is zero at $t=0$ and perpendicular to $\gamma^{\prime}(t)=T$, we have for $t \in(0,1],\left\langle\bar{\nabla}_{T} X, X\right\rangle_{N} /\langle X, X\rangle_{N} \geq \mu($ alt $)$, where $l=\|T\|_{N}$ and

$$
\mu(\text { alt })= \begin{cases}\text { al } \cot (\text { alt }) & \text { if } \max _{\gamma} K=a^{2} \text { and } l<\pi / a, \\ t^{-1} & \text { if } \max _{\gamma} K=0 \\ \text { al coth }(\text { alt }) & \text { if } \max _{\gamma} K=-a^{2} .\end{cases}
$$

From now on, for the sake of economy of notations, we will use the same symbol $\langle$,$\rangle instead of \langle,\rangle_{M},\langle,\rangle_{N}$ and $\langle,\rangle_{E}$.

Let $Q \in N$ be the center of the normal ball $B_{R}$. Let $Y_{P}$ be a unit vector tangent to $M$ at a point $P \in M$ such that $f_{*} Y_{P} \neq 0$ and $\gamma(u)$ be a geodesic in $M$ with $\gamma(0)=P$ and $\gamma^{\prime}(0)=Y_{P}$. Let $\gamma^{\prime}(u)=Y$. We join $Q$ with the point $f(\gamma(u))$ by the unique geodesic $\sigma(t, u), 0 \leq t \leq 1$, parametrized proportionally to the arc length, such that $\sigma(0, u)=Q$ and $\sigma(1, u)=f(\gamma(u))$. Put $T=\sigma_{*} \partial / \partial t, X=\sigma_{*} \partial / \partial u$ $\left(X(0, u)=0, X(1, u)=f_{*} Y\right)$. It is easy to see that $\bar{\nabla}_{X} T=\bar{\nabla}_{T} X$ and $X$ is a Jacobi field along every geodesic $t \rightarrow \sigma(t, u)$.

Now, we consider the smooth function $\Phi$ on $M$ defined by

$$
\Phi(P)=(1 / 2)\{\operatorname{dist}(Q, f(P))\}^{2} \text { for any } P \in M .
$$

We wish to estimate the gradient and the Laplacian of $\Phi$.

LEMMA 3. The following are valid: $\langle\operatorname{grad} \Phi, Y\rangle=\left\langle f_{*} Y, T\right\rangle$ and

$$
\Delta \Phi=\langle\tau(f), T\rangle+\sum_{i}\left\langle f_{*} Y_{i}, \bar{\nabla}_{T}\left(f_{*} Y_{i}\right)\right\rangle
$$

PROOF OF THE LEMMAS. The proofs of Lemmas 1 and 2 are known. Lemma $\mathbf{1}$ has been proved in [3] and it is essentially Omori's Theorem $\mathrm{A}^{\prime}$ in [4]. Lemma 2 can also be deduced from the Rauch comparison theorem given in [1, p. 32]. For the proof of Lemma 3 if we put

$$
F(\gamma(u))=\operatorname{dist}(Q, f(\gamma(u)))=\int_{0}^{1}\langle T, T\rangle^{1 / 2} d t
$$


then we get $(F \circ \gamma)^{\prime}=\langle T, T\rangle^{-1 / 2}\left\langle f_{*} Y, T\right\rangle$. So,

$$
\langle\operatorname{grad} \Phi, Y\rangle=(\Phi \circ \gamma)^{\prime}=(1 / 2)\left\{(f \circ \gamma)^{2}\right\}^{\prime}=\left\langle f_{*} Y, T\right\rangle \text {. }
$$

Also, for the Hessian form $\nabla^{2} \Phi$ of $\Phi$, we have

$$
\begin{aligned}
\nabla^{2} \Phi(Y, Y) & =Y Y \Phi-\left(\nabla_{Y} Y\right) \Phi=(\Phi \circ \gamma)^{\prime \prime}=Y\left\langle f_{*} Y, T\right\rangle \\
& =\left\langle\bar{\nabla}_{Y}\left(f_{*} Y\right), T\right\rangle+\left\langle f_{*} Y, \bar{\nabla}_{Y} T\right\rangle=\langle L(Y, Y), T\rangle+\left\langle f_{*} Y, \bar{\nabla}_{T}\left(f_{*} Y\right)\right\rangle .
\end{aligned}
$$

So, the second relation of Lemma 3 follows from the above because $\Delta \Phi$ is the trace of $\nabla^{2} \Phi$.

3. Proof of the theorem. The vector field $X$ can be written $X=X_{K}+$ $\left(\langle X, T\rangle / l^{2}\right) T$, where $X_{K}$ is normal to $T$. If $R$ is the curvature tensor of $N$ we have

$$
T^{2}\langle X, T\rangle=\left\langle\bar{\nabla}_{T} \bar{\nabla}_{T} X, T\right\rangle=\langle R(T, X) T, T\rangle=0 .
$$

So, $T\langle X, T\rangle=h(u)$ and $\langle X, T\rangle=h(u) t$. From the above relations we get, at $t=1$

$$
\left\langle X_{K}, X_{K}\right\rangle=\left\langle f_{*} Y, f_{*} Y\right\rangle-\frac{\left\langle f_{*} Y, T\right\rangle^{2}}{l^{2}}
$$

and

$$
\left\langle\bar{\nabla}_{T}\left(f_{*} Y\right), f_{*} Y\right\rangle=\left\langle\bar{\nabla}_{T} X_{K}, X_{K}\right\rangle+\frac{1}{l^{2}}\left\langle f_{*} Y, T\right\rangle^{2} .
$$

It is easy to see that $X_{K}=X-\left(h(u) t / l^{2}\right) T$ is a Jacobi field, normal along every geodesic $t \rightarrow \sigma(t, u)$ with $X_{K}(0)=0$. So, applying Lemma 2 for $X_{K}$, we have at $t=1$

$$
\frac{\left\langle\bar{\nabla}_{T} X_{K}, X_{K}\right\rangle}{\left\langle X_{K}, X_{K}\right\rangle} \geq \mu(a l)>0 .
$$

Now, we pick a point $P_{0} \in M$ such that $f\left(P_{0}\right) \neq Q$ and we put $l_{0}=\operatorname{dist}\left(Q, f\left(P_{0}\right)\right)$. Then applying Lemma 1 for the function $\Phi$ we have that for any $\varepsilon^{\prime}>0$ and $\varepsilon>0$ there exists a point $P \in M$ depending on $P_{0}$ such that

$$
\begin{aligned}
& \text { (a) } l=\operatorname{dist}(Q, f(P)) \geq \operatorname{dist}\left(Q, f\left(P_{0}\right)\right)=l_{0} \text {; } \\
& \text { (b) }\|\operatorname{grad} \Phi\|(P)<\varepsilon^{\prime} ; \\
& \text { (c) } \Delta \Phi(P)<\varepsilon
\end{aligned}
$$

We are going to use the relations (1)-(4) for this point $P$. From (1) using Lemma 3 and $(4)(a),(b)$, we deduce now

$$
\left\langle X_{K}, X_{K}\right\rangle>\left\langle f_{*} Y, f_{*} Y\right\rangle-\frac{\varepsilon^{\prime 2}}{l_{0}^{2}} .
$$

When $\mu(a l)-1 \geq 0$ from (2) using (1), (3) and (5), we obtain

$$
\begin{aligned}
\left\langle\bar{\nabla}_{T}\left(f_{*} Y\right), f_{*} Y\right\rangle & =\left\langle f_{*} Y, f_{*} Y\right\rangle-\left\langle X_{K}, X_{K}\right\rangle+\left\langle\bar{\nabla}_{T} X_{K}, X_{K}\right\rangle \\
& \geq\left\langle f_{*} Y, f_{*} Y\right\rangle-\left\langle X_{K}, X_{K}\right\rangle+\left\langle X_{K}, X_{N}\right\rangle \mu(a l) \\
& >\left\langle f_{*} Y, f_{*} Y\right\rangle+(\mu(a l)-1)\left(\left\langle f_{*} Y, f_{*} Y\right\rangle>-\varepsilon^{\prime 2} / l_{0}^{2}\right) .
\end{aligned}
$$

So,

$$
\left\langle\bar{\nabla}_{T}\left(f_{*} Y\right), f_{*} Y\right\rangle>\left\langle f_{*} Y, f_{*} Y\right\rangle \mu(a l)-\varepsilon^{\prime 2}(\mu(a l)-1) / l_{0}^{2} .
$$


If $f_{*} Y_{P}=0$ for some $Y_{P} \in T M$ then the last inequality is trivial. So, applying (6) to an orthonormal basis $Y_{i}$, adding and using Lemma 3, we get

$$
\Delta \Phi(P)>\langle\tau(f), T\rangle+\sum_{i}\left\langle f_{*} Y_{i}, f_{*} Y_{i}\right\rangle \mu(a l)-\varepsilon^{\prime 2}(\operatorname{dim} M)(\mu(a l)-1) / l_{0}^{2} .
$$

Given any positive integer $m$ we choose $\varepsilon=1 / m$ and $\varepsilon^{\prime 2}=\varepsilon l_{0}^{2} / \operatorname{dim} M$. Using (4)(c) for the points $P_{m}$, which correspond to $m$, the last inequality is converted into

$$
\frac{1}{m}>\Delta \Phi\left(P_{m}\right)>\langle\tau(f), T\rangle+e(f) \mu\left(a l_{m}\right)-\frac{1}{m}\left(\mu\left(a l_{m}\right)-1\right)
$$

where $l_{m}=\operatorname{dist}\left(Q, f\left(P_{m}\right)\right)$. So, if we suppose that the theorem is not valid, hence $\tau(f) \equiv 0$, we get $e(f)<1 / m$, which contradicts the hypothesis of the theorem.

When $\mu(a l)-1<0$, from (2) using (3) and (1), we have

$$
\left\langle\bar{\nabla}_{T}\left(f_{*} Y\right), f_{*} Y\right\rangle \geq \mu(a l)\left\langle f_{*} Y, f_{*} Y\right\rangle .
$$

For the rest of the proof we work as before.

An immediate consequence of this theorem is the following corollary.

COROLlary. If $f: M \rightarrow N$ is a harmonic map with $e(f) \geq c>0$, then $f(M)$ is not contained in a normal ball $B_{R}\left(R<\pi / 2 \sqrt{K_{0}}\right.$ if $\left.K_{0}>0\right)$.

If $N$ is a Hadamard manifold (i.e. a simply-connected complete Riemannian manifold with nonpositive sectional curvature) then the corollary says: For any harmonic map $f: M \rightarrow N$ with $e(f) \geq c>0$ the image $f(M)$ is unbounded.

\section{REFERENCES}

1. J. Cheeger and D. G. Ebin, Comparison theorems in Riemannian geometry, North-Holland Math. Library, vol. 9, North-Holland, Amsterdam, 1975.

2. J. Eells, Jr. and L. Lemaire, A report on harmonic maps, Bull. London Math. Soc. 10 (1978), 1-68.

3. Th. Hasanis and D. Koutroufiotis, Immersion of bounded mean curvature, Arch. Math. 33 (1979), 170-171.

4. H. Omori, Isometric immersions of Riemannian manifolds, J. Math. Soc. Japan 19 (1967), 205214.

Department of MAThematics, University of IOANNinA, IoANnina, GReECE 